\title{
Comparing the Prevalence of Chronic Pain After Sternotomy in Patients Undergoing Coronary Artery Bypass Grafting Using The Internal Mammary Artery and Other Open Heart Surgeries
}

\author{
Hamid Kamalipour $^{1, *}$; Ali Vafaei $^{2}$; Asef Parviz Kazemi ${ }^{2}$; Saeed Khademi ${ }^{2}$ \\ ${ }_{1}^{1}$ Laparoscopy Research Center, Shiraz University of Medical Sciences, Shiraz, Iran \\ ${ }^{2}$ Anesthesiology and Critical Care Research Center, Shiraz University of Medical Sciences, Shiraz, Iran \\ ${ }^{*}$ Corresponding author: Hamid Kamalipour, Laparoscopy Research Center, Shiraz University of Medical Sciences, Shiraz, IR Iran. Tel: +98-917111112, Fax: +98-7112318072, , E-mail: kama- \\ lih@yahoo.com
}

Received: February 8, 2014; Revised: May 15, 2014; Accepted: May 24, 2014

\begin{abstract}
Background: The prevalence of chronic postoperative pain after cardiac surgery has been reported from $17 \%$ to $56 \%$.
Objectives: We aimed to compare the prevalence of postoperative pain between patients who had undergone CABG using the internal mammary artery (IMA) and those who had undergone other cardiac surgeries including CABG using the saphenous vein or cardiac valvular surgeries.

Patients and Methods: In this cohort study, medical records of 188 patients were evaluated and divided into two equal groups (94 in each group); patients who had undergone CABG using the IMA (IMA group) and those who had undergone other cardiac surgeries using the saphenous vein or other cardiac valvular surgeries (non-IMA group). The patients' data were recorded in a self-structured questionnaire and then phone interviews were performed 3 months after the operations regarding the rate of postoperative pain. The severity of chronic pain was rated based on the numerical rating pain scale.

Results: The two groups differed significantly regarding the prevalence of pain ( $\mathrm{P}=0.023)$. In the IMAgroup, 83(88.3\%)patients experienced pain lasting for more than three months compared to 71 (75.5\%) patients in non-IMA group. The two groups differed significantly with respect to the severity of chronic pain after cardiac surgery via sternotomy $(\mathrm{P}=0.001)$. The groups did not differ significantly regarding the effects of chronic pain on their sleep, referral to a physician, and drug consumption to alleviate their pain. The IMA group experienced more complications at work and during their occupational activity.

Conclusions: The rate and severity of chronic pain after cardiac surgery via sternotomy was higher in patients undergoing CABG with separation of IMA for revascularization.
\end{abstract}

Keywords:Chronic Pain; Cardiac Surgery; Sternotomy; Internal Mammary Artery

\section{Background}

According to the International Association for the Study of Pain (IASP), chronic pain is a pain without any determined biological rate which persists after the normal rehabilitation period of a tissue for about three months (1). Despite fundamental developments in understanding pain mechanisms and treatments, postoperative chronic pain is still in an undesirable state (2) the importance of this issue was determined for the first time in 1992 in a study on patients referring to a pain clinic in Scotland. In this study, $20 \%$ of the patients stated that surgery was the leading factor affecting their pain as well as the only cause of pain in $50 \%$ of the cases (2). Studies show that inadequate pain relief after surgeries can have negative physiological and psychological consequences, delay recovery, interfere with normal organ function, and ultimately lead to increased mortality and morbidity rates $(2,3)$.
Cardiac surgery is a potentially stressful process for patients since they are afraid of the final outcome of surgery, and believe that if they survive, sustained postoperative pain would affect and limit their routine life (4). Similar to any invasive technique used in cardiac surgery, open heart surgery with the sternotomy approach is painful. Trauma and distributed tissue damage increase during cardiac surgery leading to the release of inflammatory mediators from dead or damaged cells which play a role in creating pain (4). Based on previous studies chronic chest pain after cardiac surgery via sternotomy is a serious problem for $17-56 \%$ of patients (5). Moreover, patients undergoing coronary artery bypass grafting (CABG) by the separation of the internal mammary artery (IMA) express more severe pain after surgery (6). During cardiac surgery with the separation of the IMA, soft tissues and

Implication for health policy/practice/research/medical education:

Cardiac surgery is a potentially stressful process for patients. We aimed to compare the prevalence of postoperative pain between the two groups of patients; those who had undergone CABG using the IMA (case group) and patients who had undergone other cardiac surgeries including CABG using the saphenous vein or cardiac valvular surgeries (control group) to understand, follow, and treat factors leading to chronic postoperative pain.

Copyright (C) 2014, Iranian Society of Regional Anesthesia and Pain Medicine(ISRAPM); Published by KowsarCorp. This is an open-access article distributed under the terms of the Creative Commons Attribution License, which permits unrestricted use, distribution, and reproduction in any medium, provided the original work is properly cited. 
nerves (intercostal and brachial nerve network) are damaged. This damage could be caused by the asymmetrical and prolonged traction of the opened sternal halves leading to higher brachial network damage and consequently chronic and sustained pain after surgery (7).

\section{Objectives}

Considering the importance of this issue, we aimed to compare the prevalence of postoperative pain between the two groups of patients; patients who had undergone CABG using the IMA (IMA group) and those who had undergone other cardiac surgeries including CABG using the saphenous vein or cardiac valvular surgeries (nonIMA group) to understand, follow, and treat factors leading to chronic postoperative pain.

\section{Patients and Methods}

In this cohort study, medical records of 188 patients referred to Nemazee and Shahid Faghihi Hospitals, Shiraz, Southern Iran during 2010-2012 were evaluated and divided into two equal groups (94 in each group); patients who had undergone CABG using the IMA (IMA group) and those who had undergone other cardiac surgeries including CABG using the saphenous vein or cardiac valvular surgeries (non-IMA group). The patients were in classes II and III of the American Society of Anesthesiologists (ASA) physical status class. The sample size was calculated using previous studies ( $\mathrm{P} 1=15 \%, \mathrm{P} 2=35 \%, \alpha=5 \%, 1-\mathrm{B}=90 \%$ ) as 94 patients in each group. The patients' data were recorded in a self-structured questionnaire and then phone interviews were performed regarding the rate of postoperative pain. The interviews were performed by an anesthesiology resident three months after the operations. The severity of chronic pain was rated based on the numerical rating pain scale (NRS) on a scale of $0-10(0=$ no pain, 1-3 = mild, 4-6 = moderate, and 7-10 = severe pain). General anesthesia was induced using the standard narcotic-based protocol for all the patients: midazolam, fentanyl, morphine, sodium thiopental for anesthesia induction and pancuronium for paralysis and maintained with isoflurane and fentanyl. Otherwise the patient was excluded. The sternum was incised using classic midline sternotomy in all patients and hypothermia during operation was in the range of $34-36{ }^{\circ} \mathrm{C}$. Pain management was similar for all patients (diclofenac suppository and/ or intravenous pethidine) in ICU. We included 25-65 yearold patients who had undergone cardiac surgery via sternotomy at least three months prior to the study, with an ejection fraction of more than $40 \%$. The exclusion criteria were as follows: redosurgery, kidney and liver failure, malignant neoplasms, alcoholism, chronic psychological problems needing medication, ICU stay of more than 5 days due to respiratory complications and wound infection, addiction, and death. Patients with incomplete records or those unwilling to cooperate or were unreachable phone interviews were also excluded and replaced.
Data was analyzed using SPSS software, version 19 (SPSS Inc., Chicago, Illinois). Chi-square, T-tests and Pearson's correlation coefficient were used where necessary.

\section{Results}

The mean \pm SD ages of the participants in the IMA and non-IMA groups were $54.5 \pm 7.3$ and $46.28 \pm 14.2$ years (range: 25-65 years), respectively. The patients' demographic variables are summarized in Table 1. The operations length ranged from 3 to 5 hours. Based on Pearson's correlation coefficient, no significant difference was found between age and severity of pain in the both groups. The postoperative ICU stay was similar in both groups and ranged from 2-5 days with a maximum of 3 days. In our study, 140 (74.5\%) patients did not have diabetes mellitus and 48 (25.5\%) had this disease. We found a significant difference between the mean \pm SD pain scores in patients with and without diabetes mellitus $(\mathrm{P}=$ 0.006). Moreover, we only found a significant difference between the mean pain scores of patients with and without diabetes in the IMA group $(P=0.01)$ but not in the non-IMA group $(\mathrm{P}=0.8)$. With respect to sex, no significant difference was found between the mean pain scores of men and women in the IMA $(\mathrm{P}=0.06)$ and non-IMA ( $\mathrm{P}$ $=0.58)$ groups. Table 2 shows the mean \pm SD pain scores in both groups with respect to some variables. The two groups differed significantly regarding the prevalence of pain $(\mathrm{P}=0.023)$. In the IMA group, $83(88.3 \%)$ patients experienced pain lasting for more than three months compared to 71 (75.5\%) patients in the non-IMA group. The IMA group experienced more pain in all different positions (rest, standing, bending forward, mounting stairs, deep breathing or coughing, during housework) compared with the non-IMA group (Table 3 ). In both groups most patients had moderate chronic pain after cardiac surgery via sternotomy (66\% in the IMA group vs. $41.5 \%$ in the nonIMA group). Severe pain was experienced in 9 (9.6\%) and $3(3.2 \%)$ patients in the IMA and non-IMA groups, respectively. The two groups differed significantly regarding the severity of chronic pain after cardiac surgery via sternotomy ( $\mathrm{P}=0.001)$. The two groups did not differ significantly regarding the effects of chronic pain on their sleep, referral to a physician, and drug consumption to alleviate their pain. However, the IMA group experienced more complications at work and during their occupational activity compared with the non-IMA group $(\mathrm{p}=0.001$, Table 4). Moreover, pain in sites other than the site of operation (shoulders, neck, and upper extremities) was more prevalent in the IMA group compared with the non-IMA group $(\mathrm{P}<0.001$, Table 5). We also found a significant difference between the two groups regarding localized pain at surgical site or being distributed throughout the chest $(\mathrm{P}<$ 0.001). In the IMA group, 30 (31.9\%) patients had pain at the site of surgery, while 56 (59.6\%) had distributed pain throughout the chest. The corresponding figures for the non-IMA group were 48 (51.1\%) and 23 (24.5\%), respectively. 
Kamalipour H et al.

Table 1. Comparison of Frequency (\%) and/or Mean \pm SD of Some Related Variables in The IMA and Non-IMA Groups

\begin{tabular}{|c|c|c|}
\hline Variable & IMA Group & Non-IMA Group \\
\hline Ejection Fraction 50-60\% & $53(56.4)$ & $54(57.4)$ \\
\hline \multicolumn{3}{|l|}{ Diabetes Mellitus } \\
\hline Yes & $35(37.2)$ & $13(13.8)$ \\
\hline No & $59(62.8)$ & $81(86.2)$ \\
\hline \multicolumn{3}{|l|}{ Sex } \\
\hline Male & $55(58.5)$ & $41(43.6)$ \\
\hline Female & $39(41.5)$ & $53(56.4)$ \\
\hline \multicolumn{3}{|l|}{ History of Smoking } \\
\hline Yes & $27(28.7)$ & $15(16)$ \\
\hline No & $67(71.3)$ & $79(84)$ \\
\hline
\end{tabular}

Table 2. Mean \pm SD Pain Scores in the IMA And Non-IMA Groups Regarding Some Variables

\begin{tabular}{|c|c|c|c|c|}
\hline Variable & IMA Group & Non-IMA Group & Mean \pm SD & PValue \\
\hline Diabetes Mellitus & & & & 0.006 \\
\hline Yes & $5.02 \pm 1.48$ & $3 \pm 1.68$ & $4.74 \pm 1.77$ & \\
\hline No & $4.05 \pm 1.88$ & $3.1 \pm 2.2$ & $3.53 \pm 2.1$ & \\
\hline Sex & & & & 0.41 \\
\hline Male $(n=96)$ & $4.14 \pm 2.09$ & $3 \pm 2.4$ & $3.65 \pm 2.29$ & \\
\hline Female $(n=92)$ & $4.79 \pm 1.21$ & $3.24 \pm 1.91$ & $3.9 \pm 1.81$ & \\
\hline History of Smoking & & & & 0.42 \\
\hline $\operatorname{Yes}(n=42)$ & $4.33 \pm 2.27$ & $3.4 \pm 2.22$ & $4 \pm 2.27$ & \\
\hline No $(n=146)$ & $4.44 \pm 1.59$ & $3.08 \pm 2.12$ & $3.71 \pm 2.01$ & \\
\hline
\end{tabular}

Table 3. Frequency (\%) of Patients Experiencing Pain in Different Positions

\begin{tabular}{lccc}
\hline Position in Which Pain is Intensified & IMA Group & Non-IMA Group & P Value \\
\hline Rest & $25(26.6)$ & $8(8.5)$ & 0.001 \\
Standing & $28(29.8)$ & $8(8.5)$ & $<0.001$ \\
Bending Forward & $71(75.5)$ & $44(46.8)$ & $<0.001$ \\
Mounting Stairs & $80(85.1)$ & $66(70.2)$ & 0.014 \\
Deep Breathing or Coughing & $75(79.8)$ & $51(54.3)$ & $<0.001$ \\
During House Work & $81(86.2)$ & $64(68.1)$ & $<0.003$ \\
\hline
\end{tabular}

Table 4. Comparison of the Effect of Chronic Pain on Different Variables Between the Two Groups (Frequency \%)

\begin{tabular}{lccc}
\hline Variable & P Value & Non-IMA Group & IMA Group \\
\hline Sleep Disorders & 0.1 & $10(10.6)$ & $18(19.1)$ \\
Referral to a Physician During the Past Three Months & 0.17 & $1(1.1)$ & $4(4.3)$ \\
Drug Consumption During the Past Two Months & 0.57 & $8(8.5)$ & $6(6.4)$ \\
Occupational Disorders & 0.001 & $16(17)$ & $42(44.7)$ \\
\hline
\end{tabular}

\begin{tabular}{|c|c|c|c|}
\hline Site & IMA Group, Frequency (\%) & Non-IMA Group, Frequency (\%) & P Value \\
\hline Shoulders & $67(71.3)$ & $44(46.8)$ & 0.001 \\
\hline Neck & $58(61.7)$ & $24(25.5)$ & $<0.001$ \\
\hline Upper Extremities & $42(44.7)$ & $16(17)$ & $<0.001$ \\
\hline
\end{tabular}




\section{Discussion}

We found that chronic pain after cardiac surgery was more prevalent in the IMA group (88.3\%) than the nonIMA group (75.5\%). This percentage was considerably higher than other related studies, which could be attributed to different surgical techniques during sternotomy and separating the IMA, duration of operation, patient's position, and postoperative acute pain management. In previous studies, chronic postoperative pain after cardiac surgery through sternotomy has been reported as 30\% (3) and 56\% after CABG (8). Moreover, studies reported a $20.6 \%$ brachial damage during cardiac surgery using the $\operatorname{IMA}(9,10)$. Factors that create or intensify chronic pain after cardiac surgery were assessed in the two study groups in 6 different positions. Postoperative pain was more prevalent in the IMA group in all six positions. In another study performed on patients who had undergone $\mathrm{CABG}, 72 \%$ of patients reported pain that interfered with their daily life; (8) while, in another study $39.1 \%$ of patients reported unbearable chronic pain after the operation (11). The severity of postoperative pain was evaluated using the NRS. The prevalence of severe chronic postoperative pain was $9.6 \%$ and $3.2 \%$ in the IMA and nonIMA groups, respectively. Moderate pain was observed in $66 \%$ and $41.5 \%$ of the patients in the IMA and non-IMA groups, respectively. The corresponding figures for mild postoperative pain in the IMA and non-IMA groups were $16 \%$ and $30.9 \%$, respectively. In a study on postoperative chronic pain after cardiac surgery via sternotomy mild, moderate, and severe pain was reported as $14 \%, 1 \%$, and $2 \%$ (6). We did not find a significant difference between the IMA and non-IMA groups regarding the rate of sleep disorder and amount of consumed medication to alleviate pain. In total, $44.7 \%$ and $17 \%$ of the patients in the IMA and non-IMA groups reported that their pain interfered with their occupation. Regarding the rate of pain in sites other than the site of operation, we found that the IMA group experienced pain in shoulders, neck, and upper extremities more than the non-IMA group. In another study on chronic pain after cardiac surgery via sternotomy, the rate of shoulder, neck and back pain was lower than our study (6). We found that localized chronic pain at the site of sternum wound was lower in the IMA group while distributed chronic pain in chest was higher in this group compared with the non-IMA group. In a previous study, researchers reported a prevalence of $29 \%$ for pain at surgical sites and a prevalence of $25 \%$ for pain at the sternotomy site (6). Considering the high prevalence of chronic pain in the IMA group (88\%) and the increasing rate of cardiac surgeries using the IMA, it is necessary to follow patients before, during, and after the operation and present methods to minimize their pain experience. Several approaches have been mentioned to reduce peripheral nerve damage, especially to the brachial network, as a factor for creating chronic pain after the operation, as follows: (12) median sternotomy should be performed correctly, caudal placement of the retractor, preventing pro- longed or asymmetrical traction of the opened sternal halves, using asymmetrical traction retractors with more caution (9), and the arms should be held up for separating the IMA to prevent traction to the brachial network (12-14). Recently, by presenting methods for the separation of the IMA with thoracoscopy as well as performing CABG using less invasive methods, high sternum traction is prevented (13). Moreover, establishing a service center for controlling acute pain to identify, follow, and treat acute postoperative pain as a factor for creating chronic pain is recommended. Considering the high prevalence of chronic postoperative pain after cardiac surgery via sternotomy in cases using the IMA, the timely and early identification of effective factors and the patients at risk, facilitates the treatment of chronic pain (13). Moreover, postoperative chronic chest pain should be described for high risk patients to increase their awareness (14). Our knowledge regarding the etiology, prevention, and treatment of chronic chest pain is limited and more studies should be performed in this regard (13).

This study had some limitations such as incomplete patients' records, lack of patient's cooperation, and being unable to reach patients. In conclusion, the rate and severity of chronic pain after cardiac surgery via sternotomy was higher in the IMA group compared to the nonIMA group. It is necessary to present methods to reduce chronic pain in such patients $(15,16)$.

\section{Acknowledgments}

This article is the result of a thesis submitted to the school of medicine in partial fulfillment of the requirements for the degree of anesthesia specialty This study was also funded by a number 90-01-01-3778 from ViceChancellery of Research and Technology in Shiraz University of Medical Sciences, Shiraz, Iran.

\section{References}

1. Joshi GP, Girish P, Ogunnaike BO, Babatunde O, gunnaike Joshi.. Concequences of inadequate post operative pain relief and chronic persistent post operative pain. Anesthesiology. 2005;23:21-6.

2. van Gulik L, Janssen LI, Ahlers SJ, Bruins P, Driessen AH, van Boven WJ, et al. Risk factors for chronic thoracic pain af ter cardiac surgery via sternotomy. Eur J Cardiothorac Surg. 2011;40(6):1309-13.

3. Mark A, Chaney.. Postoperative pain management for the cardiac patient. Joel A..

4. Gulik Lv, Laura van G, Janssen L. I. , Linda I, Janssen , Ahlers S. J. G. M. , et al. Driessen, Boven WJV, Wim Jan van Boven, et all. Risk factors for chronic thoracic pain after cardiac surgery via sternotomy. European Journal of cardio. 2011;40:1309-13.

5. Kalso E, Mennander S, Tasmuth T, Nilsson E. Chronic poststernotomy pain. Acta Anaesthesiologica Scandinavica. 2001;45(8):935-9.

6. Eti Aslan F. Pain in cardiac surgery and the nursing approach. Turkish Journal of Thoracic and Cardiovascular Surgery. 2012;20(1):172-6.

7. Steeds CE, Charlotte ES. The anatomy and physiology of pain. 2013;31(2):49-53.

8. Vahl CF, Carl I, Muller-Vahl H, Struck E. Brachial plexus injury after cardiac surgery. The role of internal mammary artery 


\section{Kamalipour H et al.}

preparation: a prospective study on 1000 consecutive patients. J Thorac Cardiovasc Surg. 1991;102(5):724-9.

9. Sharma AD, Parmley CL, Sreeram G, Grocott HP. Peripheral Nerve Injuries During Cardiac Surgery: Risk Factors, Diagnosis, Prognosis, and Prevention. Anesthesia \& Analgesia. 2000;91(6):1358-69.

10. Chong AY, Clarke CE, Dimitri WR, Lip GY. Brachial plexus injury as an unusual complication of coronary artery bypass graft surgery. Postgrad Med J. 2003;79(928):84-6.

11. Taillefer MC, Carrier M, Belisle S, Levesque S, Lanctot H, Boisvert AM, et al. Prevalence, characteristics, and predictors of chronic nonanginal postoperative pain after a cardiac operation: a crosssectional study. J Thorac Cardiovasc Surg. 2006;131(6):1274-80.

12. Conacher ID, Doig JC, Rivas L, Pridie AK. Intercostal neuralgia associated with internal mammary artery grafting. Anaesthesia.
1993;48(12):1070-1.

13. Young GH, Ho Young G, Yun MJ, Mi Ja Y, Kim J. E. , Ji Eun K, et al. Brachial plexus injury following median sternotomy. Korean $J$ Anesthesiol. 2012;63(3):286-7.

14. Cohen AJ, Moore P, Jones C, Miner TJ, Carter WR, Zurcher RP, et al. Effect of internal mammary harvest on postoperative pain and pulmonary function. The Annals of Thoracic Surgery. 1993;56(5):1107-9.

15. Searle RD, Simpson KH. Chronic post surgical pain.[serial on the internet]. 2009. Available from: http://www.ceaccp.oxfordjournals.org..

16. Lahtinen P, Kokki H, Hynynen M. Pain after cardiac surgery: a prospective cohort study of 1-year incidence and intensity. Anesthesiology. 2006;105(4):794-800. 